\title{
PROPOSED METHODS FOR IMPERFECT ALTERNATIVE MATRIX USING THE ANP COMPARED WITH THE HARKER METHOD
}

\author{
Toshimas Ozaki* \\ Faculty of Commerce \\ Nagoya Gakuin University \\ Nagoya, Aichi, Japan \\ E-mail: ozaki@ngu.ac.jp \\ Akihiro Itoh \\ Faculty of Commerce \\ Nagoya Gakuin University \\ Nagoya, Aichi, Japan \\ E-mail: aki-itoh@ngu.ac.jp \\ Kanna Miwa \\ Faculty of Commerce \\ Nagoya Gakuin University \\ Nagoya, Aichi, Japan \\ E-mail: kmiwa@ngu.ac.jp \\ Eizo Kinoshita \\ Faculty of Urban Science \\ Meijo University \\ Kani,Gifu, Japan \\ E-mail: kinoshit@urban.meijo-u.ac.jp
}

\begin{abstract}
This paper focuses on the decision-making by the ANP by considering the alternative matrix as the imperfect matrix. The ABIA method and the P-ABA method are proposed, which have the feature by the criteria matrix based on the matrix inverse of the loss matrix, and which are the way to presume the missing values in the alternative matrix from the eigenvector. However, the ABIA method does not give a stable estimation or the same solution as the Harker method for an imperfect evaluation matrix. In the P-ABA method, the same estimates as the Harker method are stably obtained by artificially providing some information for the imperfect matrix. The essence for the decision-making is considered through this paper.
\end{abstract}

Keywords: ANP, Harker Method, eigenvector, alternative matrix

\section{Introduction}

In the AHP, it is assumed that the evaluation matrix does not have the missing value. However, the imperfect matrix is inevitable when pair comparison is difficult. Harker (1987) and Nishizawa (2005) et al. proposed the methods to presume the missing values of such as the matrix. Though we can presume the values even by the Harker method and the Nishizawa method, we hesitate which method is appropriate when the value of C.I. that Saaty defined is differ. Authors (Ozaki et al., 2009, 2010a, 2010b) have determined a method of solving the simple dilemma in the cubic imperfect matrix that had the missing values by using the ANP, and proposed to apply this method to the evaluation matrix of the AHP. This method is that

\footnotetext{
* Corresponding author
} 
the missing values in the alternative matrix can be presumed such that the criteria matrix is derived with using the inverse alternative matrix. However, the missing values cannot be presumed by this proposed method, and can be presumed by the Harker method. This proposed method has a problem of robustness as the available method. The reason the source of the problem in the application of the ANP for the imperfect alternative matrix seems that the method of the decision-making in the ANP has not been clarified yet in the mathematical principle. This paper proposes the way to address the faults of this method by resolving the decision-making of the ANP and the imperfect alternative matrix.

\section{ABIA method and its problem}

Now, for convenience, we consider the imperfect matrix $U$ with all missing elements from lower left to upper right, and the criteria matrix $\mathrm{W}$.

$$
\begin{aligned}
\boldsymbol{U} & =\left[\begin{array}{cccc}
1 & a_{12} & a_{13} & 0 \\
1 / a_{12} & 1 & 0 & a_{24} \\
1 / a_{13} & 0 & 1 & a_{34} \\
0 & 1 / a_{24} & 1 / a_{34} & 1
\end{array}\right] \\
\mathbf{W} & =\frac{1}{A}\left[\begin{array}{cccc}
a_{12} a_{13} a_{24} a_{34} & a_{12} a_{13}{ }^{2} a_{34}{ }^{2} & a_{12}{ }^{2} a_{13} a_{24}{ }^{2} & 0 \\
a_{12} a_{24}{ }^{2} & a_{12} a_{13} a_{24} a_{34} & 0 & a_{13}{ }^{2} a_{24} a_{34}{ }^{2} \\
a_{13} a_{34}{ }^{2} & 0 & a_{12} a_{13} a_{24} a_{34} & a_{12}{ }^{2} a_{24} a_{34}{ }^{2} \\
0 & a_{12}{ }^{2} a_{24} & a_{13}{ }^{2} a_{34} & a_{12} a_{13} a_{24} a_{34}
\end{array}\right] \\
0 & a_{13}{ }^{2} a_{34}{ }^{2}+a_{12} a_{13} a_{24} a_{34}+a_{12}{ }^{2} a_{24}{ }^{2}
\end{aligned}
$$

The criteria matrix $\mathrm{W}$ is defined from the imperfect alternative matrix of Eq. (1), and the eigenvector $\mathbf{z}\left(z_{1}, z_{2}, z_{3}, z_{4}\right)$ of the ANP is shown by the eigenvector of the agreement matrix UW: $\left(\sqrt{a_{12} a_{13} a_{24} a_{34}}\right.$, $\left.\sqrt{a_{12}{ }^{-1} a_{13} a_{24} a_{34}{ }^{-1}}, \sqrt[4]{a_{12} a_{13}{ }^{-1} a_{24} a_{34}{ }^{3}}, 1\right)$. The loss element $(1,4)$ and element $(2,3)$ of Eq. (1) are presumed to be $z_{1} / z_{4}=\sqrt{a_{12} a_{13} a_{24} a_{34}}$ and $z_{2} / z_{3}=\sqrt{a_{12}{ }^{-1} a_{13} a_{24} a_{34}{ }^{-1}}$ respectively. These estimates are the same as those obtained by the Harker method. We consider that the eigenvector $\mathbf{z}\left(z_{1}, z_{2}, z_{3}, z_{4}\right)$ of the agreement matrix is able to presume the missing values for the imperfect AHP, and name the ABIA method (Method of ANP Based on Inverse Alternative). However, estimate is not obtained stably by the ABIA method for an imperfect evaluation matrix of four by four as the following imperfect alternative matrix.

$$
\boldsymbol{U}=\left[\begin{array}{cccc}
1 & 0 & 5 & 0 \\
0 & 1 & 2 & 3 \\
1 / 5 & 1 / 2 & 1 & 4 \\
0 & 1 / 3 & 1 / 4 & 1
\end{array}\right], \quad \boldsymbol{U}=\left[\begin{array}{cccc}
1 & 2 & 0 & 0 \\
1 / 2 & 1 & 0 & 3 \\
0 & 0 & 1 & 4 \\
0 & 1 / 3 & 1 / 4 & 1
\end{array}\right]
$$

Therefore, we focus on an imperfect evaluation matrix of four by four, and solve the problems with the ABIA method.

\section{Proposal of P-ABIA method}

In the ABIA method, the agreement point is shown by the eigenvector of the ANP, which is composed of the imperfect alternative matrix $U$ and the criteria matrix $\mathrm{W}$ based on the inverse alternative matrix. However, an unanticipated problem seems to occur by the ABIA method because the imperfect matrix with the missing values has not been treated in the ANP. Then, we propose the revised statutes named P-ABIA method (Power Method of ANP Based on Inverse Alternative) in this section. 


\subsection{Examination of the imperfect alternative matrix with one loss in the rectangle Numerical Example 1}

We examine the ABIA method to the imperfect alternative matrix $\mathrm{U}$ with only one loss $a$ in the rectangle. Here, the loss elements are assumed to be zero because it cannot be decided.

$$
\boldsymbol{U}=\left[\begin{array}{cccc}
1 & a & a_{13} & a_{14} \\
1 / a & 1 & a_{23} & a_{24} \\
1 / a_{13} & 1 / a_{23} & 1 & a_{34} \\
1 / a_{14} & 1 / a_{24} & 1 / a_{34} & 1
\end{array}\right]
$$

In the ABIA method, $a=z_{1} / z_{2}=\sqrt{a_{13} a_{14} a_{23}{ }^{-1} a_{24}{ }^{-1}}$ is obtained from the principle eigen-vector $\mathbf{z}$ of the agreement matrix. On the other hand, when Eq. (4) is described as maximum eigenvalue $\lambda_{H}$ and eigenvector $\mathbf{y}\left(y_{1}, y_{2}, y_{3}, y_{4}\right)$ by the matrix of Harker, $\left(\lambda_{H}-2\right) y_{1}=a_{13} y_{3}+a_{14} y_{4}$ and $\left(\lambda_{H}-2\right) y_{2}=a_{23} y_{3}$ are obtained from first and second rows of the matrix. We obtain $y_{1} / y_{2}=\sqrt{a_{13} a_{14} a_{23}{ }^{-1} a_{24}{ }^{-1}}$ as the missing value after taking the geometric mean of the right sides of these equations and dividing the former equation by the latter. That is, the presumption in the alternative matrix with either of element of loss becomes the same estimates obtained by the Harker method. We substitute the missing values for Eq. (4), and assume the maximum eigenvalue of the alternative matrix to be $\lambda$. The maximum eigenvalue $\lambda$ is obtained as the function of $a$ when the first row of $U \mathrm{x}=\lambda \mathrm{x}$ is expanded.

$$
\lambda=1+\sqrt{a} \sqrt[4]{a_{23} a_{24} a_{13}{ }^{-1} a_{14}{ }^{-1}}+\sqrt[-4]{a} \sqrt[4]{a_{13}{ }^{2} a_{23}{ }^{-1} a_{34} a_{14}{ }^{-1}}+\sqrt[-4]{a} \sqrt[4]{a_{14}{ }^{2} a_{24}{ }^{-1} a_{34}{ }^{-1} a_{13}{ }^{-1}}
$$

Because of $d \lambda / d a=0, a$ becomes $\sqrt{a_{13} a_{14} a_{23}{ }^{-1} a_{24}{ }^{-1}}$. The presumed values are those in which the maximum eigenvalue of the alternative matrix with no missing values is actually assumed to be mini-mum. Therefore, the validity of the presumed values can be obtained with the C.I. of the alternative matrix with no missing values. When the imperfect alternative matrix whose rectangule loss is one piece, the same values such as the Harker method are presumed. Because the ABIA method need not be corrected, the PABIA method is based on the imperfect matrix with one piece of rectangule loss.

\subsection{Solution of no presumed values and of values different from those obtained by Harker's me- thod by the ABIA method}

For the general solution described above, the more we have the missing values, the more increased we have zeros to the element of the agreement matrix. The principal eigenvector is difficult to calculate mathematically, even with the Harker method. In addition, the P-ABIA method is judged by the C.I. which is defined by Saaty. Therefore, the presumed values by the P-ABIA method are compared with those obtained by the Harker method according to the example of the numerical values.

\section{Numerical Example 2}

The missing values to be presumed are shown as Eq. (6), and those values are presumed to be $a=3.135$, and $b=12.99$ obtained by the Harker method. Furthermore, the maximum eigenvalue of the alternative matrix for which the values are substituted is 4.083 , and the C.I. is 0.028 .

$$
\boldsymbol{U}=\left[\begin{array}{cccc}
1 & a & 5 & b \\
1 / a & 1 & 2 & 3 \\
1 / 5 & 1 / 2 & 1 & 4 \\
1 / b & 1 / 3 & 1 / 4 & 1
\end{array}\right]
$$


Because the eigenvector of the agreement matrix UW cannot be determined by the ABIA method, this case is an example in which the missing values cannot be presumed.

$$
\boldsymbol{U} \boldsymbol{W}=\left[\begin{array}{cccc}
1 & 4 & 0 & -12 \\
0 & 1 & 0 & 0 \\
1 / 5 & 4 / 5 & 1 & -12 / 5 \\
0 & 0 & 0 & 1
\end{array}\right]
$$

We can derive $\operatorname{det}(\boldsymbol{U} \boldsymbol{W}-\boldsymbol{\lambda} \boldsymbol{I})=(1-\lambda)^{4}$ as the characteristic polynomial of Eq. (6) using the eigenvalue $\lambda$ so that the eigenvalue is the multiplicity of one. However, the eigenvalue exists from first and third rows of the agreement matrix of Eq. (7) in the closed disc of $7 / 5$ in the radius in the complex plane that centers on one according to the theorem of Gershgorin, this existence of the eigenvalue is denied because of the multiplicity of one. Furthermore, the eigenvalue cannot exist from both second and forth rows of the agreement matrix of Eq. (7) because of the radius of zero in the complex plane. This is a reason why the eigenvector of Eq. (7) cannot be determined.

The missing values can be presumed by the agreement between the imperfect matrix $U$ and the criteria matrix $\mathrm{W}$ in the ABIA method, so that the agreement of both becomes difficult to obtain when zero increases to the element of the agreement matrix UW like this. That is, it is possible to agree if there is some information in $a$ or $b$ in the alternative matrix Eq. (6). Then, we propose the P-ABIA method to which some information $x$ is artificially provided to the missing value of the ABIA method, and assume that the information $x$ is uncertainty. There are two cases with $a=x$, and $b=x$ in this example.

(a) $a=x$

Because the missing value is only by artificially providing information $x$ in this case, agreement $\mathrm{UW}$ is as follows:

$$
\boldsymbol{U W}=\left[\begin{array}{cccc}
1 & 0 & 0 & \frac{60 x}{3 x+20} \\
\frac{3}{3 x+20} & 1 & 0 & \frac{60}{3 x+20} \\
\frac{4}{3 x+20} & 0 & 1 & \frac{60 x}{5(6 x+20} \\
\frac{1}{3 x+20} & 0 & 0 & 1
\end{array}\right]
$$

Because the components of the eigenvector $z$ of this agreement matrix UW can be shown to be $z_{1}=1$, $z_{2}=(\sqrt{15 x}+10) / 10 x$, and $z_{4}=1 / 2 \sqrt{15 x}$ as an analytical solution when the eigenvalue is $1+2 \sqrt{15 x} /(3 x+$ 20 ), the missing value $b$ is obtained to be $2 \sqrt{15 x}$. Therefore, the missing value becomes $b$ that can be rewitten as $2 \sqrt{15 x}$ when assuming $a=x$. We substitute these values for Eq. (6), define the eigenvector to be $\mathrm{x}$, and search for $x$ that minimizes the maximum eigenvalue of the evaluation matrix, and search for $\mathrm{x}$ that minimizes the maximum eigenvalue of the evaluation matrix. The maximum eigenvalue $\lambda$ is shown as the following equation when the first row of $U x=\lambda x$ is expanded:

$$
\lambda=1+2 /(\sqrt[4]{240})+\sqrt[4]{6} / \sqrt[4]{10 \sqrt{15}} \cdot \sqrt[8]{x^{3}}+5 * \sqrt[4]{2 / 5} / \sqrt[4]{10 \sqrt{15}} \cdot \sqrt[8]{x^{-3}}
$$

Because $d \lambda / d x=0$ which fulfills becomes $x=3.466807$, the presumed values become $a=3.4668$, and $b=14.4225$, and the validity of these values can be judged from C.I. $=0.027$.

(b) $b=x$

Because artificially providing the missing value $b$ information $x$ in this case, the eigenvector $\mathrm{z}$ of the agreement matrix UW can be shown to be $z_{1}=1, z_{2}=\sqrt{6 / 5 x}$. Therefore, the missing value becomes $a$ that can be rewritten as $\mathrm{z}_{1} / \mathrm{z}_{2}=\sqrt{5 x / 6}$ when assuming $b=x$. We substitute $a$ and $b$ for Eq. (6), and search for $\mathrm{x}$ that minimizes the maximum eigenvalue of Eq. (6). The maximum eigenvalue $\lambda$ is shown as the following equation when the first row of $U x=\lambda x$ is expanded: 


$$
\lambda=2+5 \sqrt{5^{-1}} \cdot \sqrt[4]{2 \sqrt{6 / 5}} \cdot \sqrt[8]{x^{-3}}+\sqrt[-4]{60 \sqrt{5 / 6}} \cdot \sqrt[8]{x^{3}}
$$

Because $d \lambda / d x=0$ which fulills becomes $x=14.4225$, the presumed values become $a=3.4668$, and $b=14.4225$. Though the estimation of $a$ and $b$ is higher than that obtained by the Harker method in the PABIA method, the value of C.I. is slightly lower than that obtained by the Harker method.

\section{Numerical Example 3}

In this case, missing values are three pieces $(a, b$, and $c)$. We show the imperfect matrix $\mathrm{U}$ on the left, the criteria matrix $\mathrm{W}$ in the center, and the agreement matrix on the right.

$$
\boldsymbol{U}=\left[\begin{array}{cccc}
1 & 2 & a & b \\
1 / 2 & 1 & c & 3 \\
1 / a & 1 / c & 1 & 4 \\
1 / b & 1 / 3 & 1 / 4 & 1
\end{array}\right] \quad \boldsymbol{W}=\left[\begin{array}{cccc}
1 & 0 & 0 & 0 \\
0 & 1 & 0 & 3 \\
0 & 0 & 1 & 0 \\
0 & 1 / 3 & 0 & 1
\end{array}\right] \quad \boldsymbol{W} \boldsymbol{W}=\left[\begin{array}{cccc}
1 & 0 & 0 & 6 \\
1 / 2 & 1 & 0 & 3 \\
0 & 4 / 3 & 1 & 0 \\
0 & 1 / 3 & 1 / 4 & 1
\end{array}\right]
$$

The missing values of $a, b$, and $c$ become $1.5,6$, and 0.75 obtained by the Harker method, and the maximum eigenvalue is 4 . However, their values become 1.5, 3.7308, and 1.214 obtained by the ABIA method, and the maximum eigenvalue is 4.09. The estimates obtained by the ABIA method are different from values obtained by the Harker method, and the value of C.I. is inferior. Though there is many zero in the element of the agreement matrix UW as well as the Numerical Example 2 in this case, the eigenvalue and eigenvector exist from the closed disc in the radius in the complex plane that centers on one according to the theorem of Gershgorin. Then, the next three cases are examined in the P-ABIA method as follows:

$$
\begin{gathered}
\text { Case } 1 \\
\mathrm{U}_{a}=\left[\begin{array}{cccc}
1 & 2 & 0 & x \\
1 / 2 & 1 & y & 3 \\
0 & 1 / y & 1 & 4 \\
1 / x & 1 / 3 & 1 / 4 & 1
\end{array}\right], \quad \mathrm{U}_{b}=\left[\begin{array}{cccc}
1 & 2 & x & 0 \\
1 / 2 & 1 & y & 3 \\
1 / x & 1 / y & 1 & 4 \\
0 & 1 / 3 & 1 / 4 & 1
\end{array}\right], \mathbf{U}_{c}=\left[\begin{array}{cccc}
1 & 2 & x & y \\
1 / 2 & 1 & 0 & 3 \\
1 / x & 0 & 1 & 4 \\
1 / y & 1 / 3 & 1 / 4 & 1
\end{array}\right] .
\end{gathered}
$$

\section{(a) Case 1}

The value $a$ is obtained to be $\mathrm{z}_{1} / \mathrm{z}_{3}=\sqrt{x y / 2}$ from the eigenvector $\mathrm{z}$ of the agreement matrix $\mathrm{U}_{\mathrm{a}} \mathrm{W}_{\mathrm{a}}$ when assuming $b=x$ and $c=y$. The missing values of $a, b$, and $c$ are substituted for Case1 in Eq. (12), $x$, and $y$ that minimize the maximum eigenvalue of Case 1 are searched. The eigenvector of Case 1 is assumed to be $\mathrm{x}$, and then the first and third rows $\mathrm{Ux}=\lambda \mathrm{x}$ are considered. Therefore, $2^{5} 3^{2} y=x$ is obtained from the first row as $\partial \lambda / \partial x=0$, and $3^{2} x=2^{7} y$ is obtained from the third row as $\partial \lambda / \partial y=0$. The values $x=6$, and $y=3 / 4$ are obtained from these equations, and another missing value, $a=3 / 2$, is obtained from $x$ and $y$.

\section{(b) Case 2}

The value $b$ is obtained to be $\mathrm{z}_{1} / \mathrm{z}_{4}=2 \sqrt{6 x}$ from the eigenvector $\mathrm{z}$ of the agreement matrix $\mathrm{U}_{\mathrm{b}} \mathrm{W}_{\mathrm{b}}$ when assuming $a=x$ and $c=y$. The missing values of $a, b$, and $c$ are substituted for Case 2 in Eq. (12), $x$, and $y$ that minimize the maximum eigenvalue of Case 2 are determined. The eigenvector of Case 2 is assumed to be $\mathrm{x}$, and the first and fourth rows of $\mathrm{Ux}=\lambda \mathrm{x}$ are considered. Therefore, $x^{3}=6 y^{2}$ is obtained from the first row as $\partial \lambda / \partial x=0$, and $2^{5} x y^{2}=3^{3}$ is obtained from the fourth row as $\partial \lambda / \partial y=0$. The values $x=6$ and $y=3 / 4$ are obtained from these equations, and another missing value, $a=3 / 2$, is obtained from $x$ and $y$.

(c) Case 3 
The value $c$ is obtained to be $\mathrm{z}_{2} / \mathrm{z}_{4}=\sqrt{6 x} / 4$ from the eigenvector $\mathrm{z}$ of the agreement matrix $\mathrm{U}_{\mathrm{c}} \mathrm{W}_{\mathrm{c}}$ when assuming $a=x$ and $b=y$. The eigenvector of Case 3 is assumed to be $\mathrm{x}$, and the second and third rows of $\mathrm{Ux}=\lambda \mathrm{x}$ are considered. Then, $x y^{2}=2^{1} 3^{3}$ and $3 y^{2}=2^{5} x^{3}$ are obtained from the second and thirds rows as $\partial \lambda / \partial x=0$ and $\partial \lambda / \partial y=0$. Therefore, the values $x=1.5$, and $y=6$ are obtained from these equations, and another missing value, $c=3 / 4$, is obtained from $x$ and $y$. The maximum eigenvalue of the alternative matrix for which $a, b$, and $c$ are substituted is 4 , and the C.I. is minimized. Estimates of $a, b$, and $c$ obtained by the ABIA method are corrected by the P-ABIA method.

\section{Conclusion}

The present study is a proposal of the P-ABIA method to solve the fault of the ABIA method. The following points are made clear in this paper.

(1) The solution of the agreement matrix UW which has the fault of the ABIA method is stabilized by providing some information to an imperfect matrix with the missing values.

(2) We provide the condition of minimizing the maximum eigenvalue of the matrix to it in order to specify some information. This condition is originally possessed in the Harker method.

(3) Though the eigenvector is used in the Harker method and the ABIA method, the P-ABIA method need not be used the eigenvector only by the calculation of the matrix.

(4) To provide some information to the imperfect alternative matrix is to show the compromise.

The P-ABA method yields the same estimates as the Harker method, seems to indicate the essence for the decision-making.

\section{References}

Harker, P.T. and Vargas, LG. (1987). Incomplete Pairwise Comparisons in the Analytic Hierarchy Process. Mathematical Modeling, 9. 838-848.

Nishizawa, K. (2005). Estimation of Unknown Comparisons in Incomplete AHP. Report of the Research Institute of Industrial Technology, Nihon University, 77.1-9.

Ozaki, T., Sugiura, S., and Kinoshita, E. (2009). Dissolution of the dilemma or circulation problem using the Analytic Network Process. Proceedings of the Tenth International Symposium on the Analytic Hierarchy Process 2009, Pittsburgh.PA.

Ozaki, T. and Miwa, K. (2010). An Approximation Process of Missing Value in Imperfect Evaluation Matrix. Journal of Japanese Symposium on the Analytic Hierarchy Process, 3.115-121.

Ozaki, T. and Miwa, K (2010). AN APPROXIMATION PROCESS OF MISSING VALUE IN IMPERFECT EVALUATION MATRIX. Annual Research Journal of Nagoya Gakuin, 47.67-81.

Sugiura. S. and Kinoshita. E.(2005). A Dissolution of Circular Logic with concurrent Convergence Method. Research of Urban Infomatics, Meijyo University.10.115-121. 